\title{
AUTODIAGNóSTICO Y BÚSQUEDA DE INFORMACIÓN MÉDICA ONLINE: EL CASO CHILENO
}

\section{Self-diagnosis and online health information seekers: the Chilean case}

\section{Daniel Halpern, Macarena Peña-y-Lillo, Francisco Goic, Alejandra Reinoso- Aguiló, Camila Figueroa-Zepeda y Axel Troncoso-Leiva}

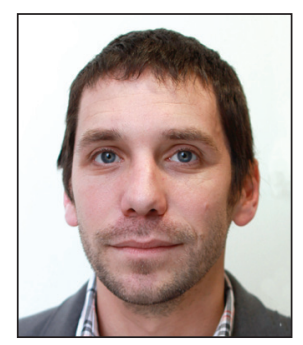

Daniel Halpern, doctor en information and communication sciences, es profesor de la Facultad de Comunicaciones de la Pontificia Universidad Católica de Chile e investigador del thinktank TrenDigital. Sus artículos han sido publicados en prestigiosas revistas académicas como Computers in human behavior y Behaviour and information technology. Su investigación se centra en las consecuencias sociales del uso de las tecnologías de información y comunicación. Ha asesorado a numerosas organizaciones y empresas en la implementación de redes sociales y desarrollo de nuevas aplicaciones. http://orcid.org/0000-0002-1569-9876

Pontificia Universidad Católica de Chile, Facultad de Comunicaciones Alameda, 340. Santiago, Chile dmhalper@uc.cl

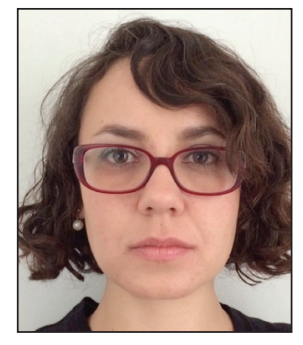

Macarena Peña-y-Lillo es periodista por la Universidad de Chile, máster en comunicación por la Universidad de Illinois at Urbana-Champaign en Estados Unidos y estudiante de doctorado en dicha institución. Su área de investigación es la comunicación de la salud, con énfasis en campañas comunicacionales y comunicación estratégica en salud, disparidades sociales en salud y comunicación, y el rol de la tecnología en la comunicación de la salud y en la salud física y psicológica de los individuos.

http://orcid.org/0000-0002-2422-571X

Universidad de Illinois at Urbana-Champaign 3001 Lincoln Hall, 702 South Wright Street, Urbana, IL 61801, Estados Unidos penayli2@illinois.edu

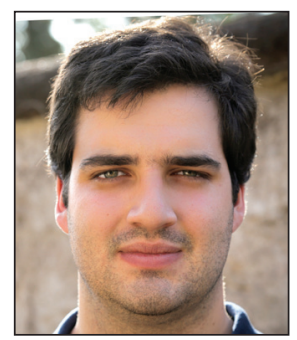

Francisco Goic es periodista e investigador centrado en temas sobre la efectividad de las políticas de salud pública. Está investigando la industria alimentaria en Chile y sus efectos sobre la población para Televisión Nacional de Chile.

http://orcid.org/0000-0002-3926-8923

Productora La Ventana Cine Rodolfo Lenz, 499. Ñuñoa, Santiago, Chile fjgoic@uc.cl

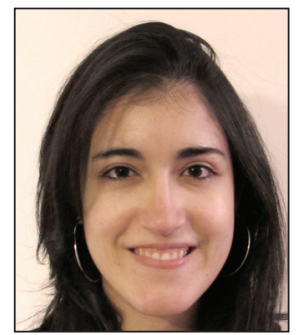

Alejandra Reinoso-Aguiló es periodista en CNN Chile. Trabaja en investigación en el área de las comunicaciones y sus áreas de interés son ética de las comunicaciones, ontología, narración de ficción y no ficción, y los alcances de internet en las relaciones sociales.

http://orcid.org/0000-0002-1257-8817 


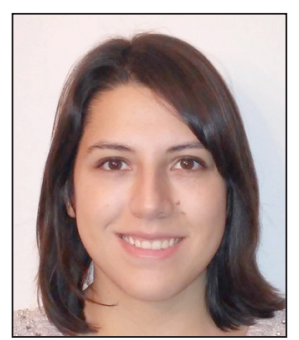

Camila Figueroa-Zepeda es periodista con minor en sociología. Investiga áreas relacionadas con el servicio público y las instituciones ya que considera que son espacios vitales para la vida de los ciudadanos que necesitan de un buen dominio y uso comunicacional.

http://orcid.org/0000-0002-9537-2506

Confederación Nacional de Funcionarios Municipales de Chile (Asemuch) Av. Suecia, 403 depto. 23, Providencia, Santiago, Chile. cpfigue1@gmail.com

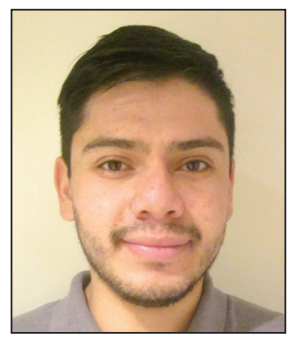

Axel Troncoso-Leiva es periodista y analista de redes sociales. Investiga temas relacionados con el alcance de internet en la sociedad y el uso de redes sociales.

http://orcid.org/0000-0002-5230-2911

Radio ADN - Iberoamericana Radio Chile Alejandro Fleming, 9797 depto. B303, Santiago, Chile. aptronco@uc.cl

\title{
Resumen
}

Se exploran los factores que inciden tanto en la búsqueda de información sobre temas de salud como en el autodiagnóstico online a través de una muestra representativa de la población con acceso a internet en Chile. Los resultados indican que tres grupos de variables explican en gran parte este fenómeno: 1) la experiencia de los usuarios al consultar a sus médicos sobre lo que encontraron online; 2 ) aspectos psicológicos-individuales como preexistencias médicas y tiempo que esperan antes de decidir ir al médico; y 3) factores tecnológicos como confianza y eficacia en el uso de internet. La investigación demostró que la búsqueda de información sobre temas de salud a través de internet es una tendencia creciente, ya que durante el último año más del $90 \%$ de los consultados dijo haber buscado al menos una vez, mientras que un $85 \%$ dijo haber revisado sus síntomas y autodiagnosticado.

\section{Palabras clave}

Internet; Salud; Salud online; Búsqueda de información; Tecnologías de información y comunicación; Información médica; Información de salud; Autodiagnóstico; Síntomas; Médicos; Pacientes; Encuestas; Chile.

\begin{abstract}
The factors that affect users as they search for health information and conduct a self-diagnosis online among a nationally representative sample of Chileans with internet access were analysed. The results indicate that three groups of variables largely explain this phenomenon: 1) the experiences that users had when commenting to their doctors about what they found online; 2) individual-psychological aspects such as pre-existing medical conditions; and 3) technological factors such as confidence and efficacy regarding internet use. Our research showed that this is a growing trend: more than $90 \%$ of respondents said that during the last year they had searched for health information on the internet at least once, while $85 \%$ said they had revised their symptoms and self-diagnosed.
\end{abstract}

\section{Keywords}

Internet; Health; E-health; Online health; Information seeking; Information and communication technologies; Health information; Self-diagnosis; Symptoms; Doctors; Patients; Surveys; Chile.

Halpern, Daniel; Peña-y-Lillo, Macarena; Goic, Francisco; Reinoso-Aguiló, Alejandra; Figueroa-Zepeda, Camila; Troncoso-Leiva, Axel (2015). “Autodiagnóstico y búsqueda de información médica online: el caso chileno". El profesional de la información, v. 24, n. 5, pp. 621-629.

http://dx.doi.org/10.3145/epi.2015.sep.10

\section{Introducción}

El objetivo de este trabajo es doble. Por una parte, entender los factores que inciden en la búsqueda de información sobre salud que pueden llevar al usuario a autodiagnosticarse online. Interesa descubrir quiénes, cómo, dónde y por qué se suman a esta preferencia, así como las plataformas que sirven para este propósito. Si bien en Sudamérica no hay investigaciones formales sobre autodiagnóstico online, estudios en Estados Unidos muestran que el $72 \%$ de los adultos estadounidenses buscan información sobre su salud en internet (Fox; Duggan, 2013).

En segundo lugar, entender el cambio que se observa en la relación médico-paciente. Diversas investigaciones han indicado que consultar información online empodera a las per- 
sonas y les da mayor independencia ante las decisiones de su médico (Donnelly; Shaw; Van-den-Akker, 2008).

Parece relevante estudiar ambos aspectos por varias razones:

- La bibliografía ha detectado una serie de riesgos que podrían ser perjudiciales para quienes buscan información sobre temas de salud. Las personas que visitan sitios de autodiagnóstico o buscan síntomas online, tienden a desarrollar una preocupación excesiva sobre su salud denominada cybercondría (Smith et al., 2006), que puede llevar a ansiedad, temor y estrés (Nijland et al., 2008). Es más, como la mayoría carece de conocimiento médico, este temor puede llevarlos a obtener falsas conclusiones médicas: por una parte pueden creer que su condición es peor de lo que realmente es, ya que los síntomas de muchas enfermedades son comunes y pueden malinterpretarse, y por otra parte pueden concluir erróneamente que no están enfermos al ver que no presentan todos los síntomas, como en muchos casos ocurre en la población más joven con el VIH (Fernández-De-Mosteyrín et al., 2014).

- La información disponible en internet no es necesariamente cierta: como es de libre acceso y no está regulada, los sitios no cuentan siempre con información fidedigna, porque pueden publicar contenido con el objetivo de atraer audiencias, o respondiendo a intereses particulares (por ejemplo, laboratorios médicos que quieren vender sus productos).

A pesar de que hoy la información es accesible a millones de usuarios a nivel global, no puede afirmarse lo mismo sobre el conocimiento que se necesita para asimilarla

En la relación médico-paciente es relevante entender la nueva dinámica como resultado del mayor acceso a la información que tienen hoy los ciudadanos. Antes el paciente era visto como un ente pasivo que reaccionaba frente a lo que el médico le sugería al asumir -ambas partes- que su opinión era la más indicada (Elwyn et al., 2011). Sin embargo, esta asimetría informacional entre lo que el médico "sabía" y el paciente "entendía" cambió en el momento en que la información médica comenzó a estar disponible para usuarios no expertos. Diversos estudios han dado cuenta de cómo las personas se han empoderado con el mayor acceso a la información y comenzado a cuestionar las decisiones adoptadas por sus médicos (Weinhold; Gastaldi, 2015). Los pacientes contrastan la información que leen en internet con la opinión del médico, lo que les ha llevado a rechazar con mayor frecuencia la opinión del profesional (Finch et al., 2008), ya que los médicos dejaron de ser percibidos como la única fuente experta (Lowrey; Anderson, 2006).

Este estudio busca dar cuenta de los cambios y diferencias que la búsqueda de información y el autodiagnóstico han producido en la relación entre médico y paciente. Para lograr este propósito, primero se revisarán las distintas variables que podrían incidir en la búsqueda de información y el autodiagnóstico en la bibliografía del área, luego se presentará el método y datos utilizados para estudiar potenciales asociaciones, y por último se discutirán las implicaciones y limitaciones del estudio, para posteriormente dar pie a futuras direcciones de investigación.

\section{Revisión bibliográfica}

\section{Búsqueda de información de salud}

Se entiende por conductas de búsqueda de información de salud los esfuerzos activos para obtener información sobre salud que van más allá de los patrones normales de exposición a contenidos a través de medios de comunicación o fuentes interpersonales (Niederdeppe et al., 2007). Han sido relacionadas con la adopción de conductas saludables como la realización de exámenes para detectar enfermedades (Hornik et al., 2013). A través de la búsqueda de información las personas pueden aprender a reconocer sus síntomas y calibrar la gravedad del problema que las aqueja. Internet se ha transformado así en una de las fuentes de información de salud por excelencia (Weinhold; Gastaldi, 2015). A diferencia de los patrones normales de exposición con que las personas consumen información en otros medios, internet permite ir más allá del "escaneo", pues implica una búsqueda activa e intencionada de información (Niederdeppe et al., 2007). Sin embargo, además de los beneficios expuestos, la bibliografía ha reconocido diversos problemas por la gran cantidad de contenido que circula en la Web, y señala la necesidad de que existan una serie de condiciones para obtener resultados positivos en este proceso.

\section{Eficacia y experiencia web}

Si bien en sus inicios la penetración de internet en Latinoamérica era reducida, hoy ha superado el $50 \%$ en gran parte de los países del Cono Sur. En Chile por ejemplo, el 60,5\% de la población tiene acceso a internet (SubTel, 2013). Si bien se han acortado las diferencias sociales, demográficas y económicas en la penetración de internet, sigue existiendo una gran brecha. A pesar de que hoy la información es accesible a millones de usuarios a nivel global, no puede afirmarse lo mismo sobre el conocimiento que se necesita para asimilarla: muchos usuarios de internet no saben dónde está la información que requieren, desconocen los mecanismos necesarios para procesarla, y más importante, carecen de las herramientas para transformarla en conocimiento específico que les permita conseguir lo que buscan (Rice, 2006).

Adicionalmente, existen diferencias con respecto al uso que se les da a las nuevas tecnologías. Diversas investigaciones señalan que personas con mayor educación tienden a usar la Web para acceder a información o utilizar servicios, mientras que aquellos con menor nivel educacional prefieren las actividades de entretenimiento. Por ende, la brecha digital hoy no sólo está dada por el acceso a la Red, sino por la habilidad inquisitiva del usuario para encontrarla y su capacidad para interpretarla correctamente.

Un ejemplo claro son los buscadores: la mayor parte de usuarios de internet cuando necesita buscar información en el área médica lo hace a través de motores de búsqueda como Google. Esto puede llegar a convertirse en una experiencia poco agradable para quienes buscan encontrar res- 
puestas a sus preguntas de salud, dada la gran cantidad de información descontextualizada o irrelevante que hay en la Red. Si no se cuenta con la experiencia necesaria, podría confundirse publicidad con información, foros o experiencias de terceros con recomendaciones médicas, o simplemente podría existir una incapacidad por parte del usuario para encontrar lo requerido. Se espera que las personas que posean un mayor grado de eficacia en el uso de la Web puedan conseguir también un mayor provecho de la información que encuentren, y así obtener mayor información de utilidad. Esto aumentará su nivel de eficacia para seguir buscando información que pueda afectar su toma de decisiones, y por tanto tendrá una tendencia mayor no sólo a buscar información online, sino también al autodiagnóstico online. Consecuentemente, se espera que:

Hipótesis 1 (H1): Los usuarios con mayor eficacia en el uso de la Web buscarán más información online sobre temas de salud y mostrarán una tasa mayor de autodiagnóstico online.

\section{Confianza en la información obtenida a través de in- ternet}

La información y los comentarios publicados en internet son evaluados constantemente por los internautas con el fin de tomar mejores decisiones. La confianza y la percepción de seguridad y responsabilidad hacia los sistemas y plataformas de autodiagnóstico online representan el $15 \%$ de los atributos que explican las conductas de búsqueda de información de salud online (Mair et al., 2012). Sin embargo, es importante destacar que estas preocupaciones pueden actuar como facilitadores o como barreras. Los ciudadanos pueden ver en el uso de las tecnologías relacionadas con el área médica una manera de reducir los errores, lo que fomentaría la captación. Por otro lado, los problemas de seguridad podrían socavar la confianza en los sistemas de autodiagnóstico online y obstaculizar su utilización generalizada (Mair et al., 2012). En función de lo anterior, es posible formular la hipótesis de que a mayor confianza en la información obtenida a través de internet, mayor será la tendencia a realizar búsquedas informativas sobre temas de salud y al autodiagnóstico online.

Hipótesis 2 (H2): Las personas con mayor confianza en la información médica encontrada online, buscarán más información sobre temas de salud y mostrarán una tasa mayor de autodiagnóstico online.

\section{Los e-pacientes y reacción del médico en torno a la búsqueda de información y autodiagnóstico}

La bibliografía sobre relación médico-paciente señala que casi dos tercios de pacientes ha discutido con su médico, al menos una vez, sobre información de su estado de salud que encuentran en internet (Fox; Duggan, 2013). Por esta razón se habla de "e-pacientes", personas que utilizan activamente las tecnologías digitales para buscar información sobre su salud. La mayoría de los profesionales de la salud, sin embargo, muestran escepticismo ante las supuestas ventajas de internet para la relación entre médico y paciente, ya que creen que pone en duda su criterio profesional
(Mira-Solves et al., 2010). Los médicos sienten que la búsqueda de información online agregó un nuevo rol a sus responsabilidades clínicas. Si bien la mayoría de profesionales se sienten obligados a lidiar con esta nueva realidad, no ha sido del todo bienvenida (Ahmad et al., 2006). Este cambio producido por las TICs ha planteado un nuevo escenario en la relación entre los profesionales de la salud y sus pacientes, y por lo mismo es relevante llevar a cabo investigaciones para ayudar a que tanto unos como otros se puedan adaptar a esta nueva dinámica.

\section{Se habla de los 'e-pacientes', personas que utilizan activamente las tecnologías digitales para buscar información sobre su salud}

Es por ello que este estudio, desde una dimensión exploratoria, aborda una serie de aspectos que tienen relación con el perfil de los nuevos e-pacientes. Además, se busca conocer si los médicos han incorporado las tecnologías de comunicación en su relación con sus pacientes. Por todo ello, se proponen las siguientes preguntas de investigación:

PI1 ¿Cuáles son las principales motivaciones que tienen los usuarios para buscar información sobre su salud online?

PI2 ¿Cuáles son las reacciones de los médicos ante la revelación de que se está buscando información online, según los pacientes?

PI3 ¿Cuáles son las TICs que más utilizan los médicos para comunicarse con sus pacientes?

La disposición de los e-pacientes hacia la búsqueda de información online y el autodiagnóstico también podría verse afectada por las indicaciones que reciben de sus médicos, que son la fuente experta que transmite confianza (Sundar; Knobloch-Westerwick; Hastall, 2007) y por ende podrían incidir en la actitud que los usuarios desarrollen hacia estas prácticas. De acuerdo con la bibliografía en comunicación y persuasión sobre temas médicos (Dillard; Shen, 2005), podría plantearse que las personas que hayan tenido una buena experiencia con su médico en relación con la búsqueda de información online, podrían haber sido persuadidos positivamente para seguir buscando información de internet.

De lo anterior se desprende que cuanto más positiva sea la experiencia, los pacientes reforzarían aún más esta práctica y lo más probable es que sigan buscando información online. Por otra parte, quienes han recibido una respuesta negativa de sus médicos al buscar información online, es probable que se hayan desalentado con esta práctica y reduzcan posteriormente la búsqueda y el autodiagnóstico online. Siguiendo esta lógica, se plantea que:

Hipótesis 3 (H3): Cuanto más positiva sea la experiencia con los médicos en la búsqueda de información online, mayor será la tendencia de los pacientes a seguir buscando información por internet y al autodiagnóstico. 


\section{Ansiedad por el cuidado de la salud y condiciones previas}

Otro de los factores que la bibliografía ha examinado como posible predictor en búsquedas y autodiagnóstico es la ansiedad por el cuidado de la salud. Esta ansiedad puede alcanzar el trastorno hipocondríaco, que se ha definido como "la presencia de una preocupación generalizada y no delirante, con temor a llegar a tener una enfermedad grave basada en la interpretación errónea de los síntomas corporales" (Pastorelli et al., 2011). Así, podría afirmarse que un usuario que presenta ansiedad por el cuidado de su salud tenderá a buscar la explicación a sus síntomas a través del autodiagnóstico online.

La bibliografía también indica que la ansiedad por la salud o la preocupación somática se puede explicar a partir de dos fenómenos (Fernández-Martínez; Fernández-Rodríguez, 2001):

- la convivencia con familiares descritos como notablemente preocupados por su salud;

- limitaciones a causa del estado de salud durante la infancia.

Ambas experiencias parecen ser antecedentes relevantes de las formas más intensas de ansiedad por la salud, como es la hipocondría (Fernández-Martínez; Fernández-Rodríguez, 2001). De lo anterior se desprenden dos hipótesis:

H4: Los usuarios con historiales clínicos y de enfermedades buscarán una mayor cantidad de información sobre salud y tenderán a autodiagnosticarse en mayor medida.

H5: Cuanto mayor sea la preocupación que los usuarios muestren sobre su salud, mayor será la búsqueda de información online sobre temas de salud y mostrarán una tasa mayor de autodiagnóstico online.

\section{Metodología}

Para responder las preguntas de investigación y comprobar las hipótesis planteadas, se aplicó una encuesta online a usuarios chilenos de internet. Para asegurar una muestra representativa de la población en Chile, se utilizó un panel online cuya composición se basa en la encuesta Casen $^{1}$ del año 2011.

Se consideraron tres variables:

- Sexo: hombres: 48,7\%; mujeres 51,3\%;

- Edad: 18-34: 55\%; 35-44: 20\%; 45-64: 22\%; 65+: 3\%;

- Geografía: Región Metropolitana: 47\%; Quinta Región: 11\%; Séptima Región: 10\%; otras regiones sur del país: $20 \%$; otras regiones del norte: $12 \%$.

El panel online, que cuenta con más de 30 mil usuarios, se elaboró mediante 3 mecanismos:

- se llamó aleatoriamente a 500 personas para invitarlas a participar en un panel online y luego se les envió un correo electrónico con un formulario en el que se les preguntaba su nombre, edad, sexo y dirección;

- selección aleatoria de estudiantes y funcionarios de universidades que llenaron un formulario con las mismas variables;
- un tercer grupo se ha ido expandiendo constantemente de dos formas: mediante el envío de correos masivos en el que los interesados se inscriben; y con los estudios que se realizan con empresas que las envían a sus propios empleados y bases de datos, que al final de los cuestionarios se inscriben para seguir participando.

Para este estudio en particular, el cuestionario se envió a 5.110 usuarios del panel, que se escogieron según las variables geográficas descritas anteriormente (región, edad y sexo), de los que 1.430 terminaron la encuesta, lo que representa una tasa de respuesta del $27,98 \%$. Los miembros seleccionados del panel recibieron un url con redirección a la encuesta mediante un email de invitación. La primera invitación se envió el día 8 de mayo de 2014, y las semanas posteriores fueron enviados emails recordatorios en dos oportunidades. El cuestionario fue aprobado por el Comité de Ética de la Pontificia Universidad Católica de Chile, y antes de contestar la encuesta los usuarios debían dar su consentimiento para participar en el estudio. La plataforma no permite asociar las respuestas con los nombres de los usuarios para asegurar su anonimato.

\section{Mediciones}

\section{Variables dependientes}

- Búsqueda de información de salud online: se consultó sobre el uso de cinco plataformas para buscar información sobre salud: motores de búsqueda como Google, redes sociales, páginas especializadas, foros de conversación o blogs, y Wikipedia ( $M=2,34 ; D S=1,03 ; \alpha=0,83$ ) en una escala de 6 puntos, donde $1=$ nunca he buscado y $6=$ todos los días.

- Autodiagnóstico online: se consultó si han buscado información online sobre síntomas de una enfermedad para luego corroborarla (85\% dijo que sí).

\section{Variables independientes}

- Eficacia y experiencia web: se utilizaron tres items de escalas previas (Norman; Skinner, 2006) sobre eficacia:

- "Confío en que puedo utilizar internet para tomar decisiones sobre mi salud"

- "Sé usar la información de salud que encuentro en internet", y

- "Sé encontrar recursos útiles en internet").

Se utilizó una escala de 5 puntos donde $1=$ muy en desacuerdo y $5=$ muy de acuerdo $(M=3,81 ; D S=0,77 ; \alpha=0,72)$

- Confianza de información online: se midió el nivel de confiabilidad de los usuarios en la información obtenida en las cinco plataformas antes consultadas. Se utilizó una escala de 5 puntos donde $1=$ nada de confianza y $5=$ mucha confianza ( $M=3,1 ; D S=0,75 ; \alpha=0,77)$.

- Antecedentes de salud: los participantes señalaron si tenían alguna enfermedad permanente o alguna preexistencia. Para esto se les presentó una lista con los problemas de salud más recurrentes donde podían seleccionar las enfermedades o agregar otra. Luego se formó una variable dummy (variable binaria) que reconoció aquellos usuarios con preexistencias médicas (24,86\%).

- Asertividad del médico frente a la búsqueda de informa- 
ción sobre salud online: se preguntó cómo había reaccionado su médico al decirle que habían buscado información en internet sobre el problema que los afectaba. Se creó una escala con respuestas que fueron de más negativas a más positivas, donde las tres negativas se codificaron como 1 (le sugirió no seguir buscando información, ofendido, preocupado: 16,6\%), como 2 neutral (indiferente: $19,9 \%$ ), y como 3 las positivas (le pareció una buena idea, una excelente idea, lo motivó a seguir buscando: $14,9 \%)$. Un $48,5 \%$ dijo que nunca había hablado con su médico de búsqueda de información sobre salud online.

- Preocupación por el estado de salud: los participantes señalaron en qué grado estaban de acuerdo con la frase: "Pienso constantemente sobre mi estado de salud", en base a una escala de 5 puntos donde $1=e n$ total desacuerdo y $5=$ muy de acuerdo $(M=3,5 ; D S=1,1)$.

\section{Variables de control}

- Edad: el concepto se operacionalizó con una variable continua de 4 items: entre 18 y 24 años (34\%); 25-34 (23\%); $35-44(15 \%) ; y$ mayores de 45 años (8\%).

- Sexo: masculino (41\%), femenino (59\%).

- Nivel socioeconómico: el concepto se operacionalizó con una variable continua de 6 items con el objetivo de conocer el ingreso mensual total del hogar de la persona que contesta la encuesta: Más de USD 7.000 (6,7\%); USD 5.000-7.000 (7,8\%); USD 3.000-5.000 (17,3\%); USD 1.6003.000 (24,9\%); USD 800-1.600 (26,3\%); menos de USD $800(17,4 \%)$.

Las preferencias de búsqueda se concentran en información sobre síntomas $(75,7 \%)$, seguido de la revisión de diagnósticos ya dados por el médico (35\%)

\section{Resultados}

\section{Análisis descriptivo}

Una gran parte de los usuarios en Chile con acceso a internet ha buscado información sobre su salud y se ha diagnosticado online. Más del $90 \%$ dijo haber buscado información online sobre su salud o la de otros al menos una vez durante el último año, mientras que un $85 \%$ dijo haber revisado sus síntomas y autodiagnosticado a través de internet.

Respondiendo a la primera pregunta de investigación, los resultados indican que las preferencias de búsqueda se concentran mayormente en información sobre síntomas como dolor de cabeza o dolor de pecho $(75,7 \%)$, seguido de la revisión de diagnósticos ya dados por el médico (35\%) y un $27,8 \%$ dijo revisar condiciones médicas graves como cáncer y enfermedades terminales.

Después de buscar en internet información sobre síntomas o problemas que creían que tenían:

- casi la mitad solicitó consultas presenciales con un profesional de la salud para confirmar el diagnóstico encontrado en la Web $(49,9 \%)$;
- un $28,6 \%$ dijo seguir los consejos encontrados en internet exceptuando la administración de remedios, un 4,6\% incluyendo la administración de medicamentos;

- un $8 \%$ indicó que no considera la información que aparece en la Web.

Respondiendo a la segunda pregunta de investigación, es relevante destacar que las nuevas tecnologías han abierto nuevos canales de comunicación entre médicos y pacientes. Un $43 \%$ dijo que después de alguna consulta el médico les había dicho que los contactaran utilizando algún tipo de tecnología. Las preferencias más altas fueron correo electrónico y teléfono móvil con $19,8 \%$ y $19,7 \%$ respectivamente. Whatsapp ocupó un $6 \%$ de las preferencias, mientras que el beeper (el mensáfono que tuvo su auge en los años 90) sólo alcanzó un $0,3 \%$.

Las nuevas tecnologías han abierto nuevos canales de comunicación entre médicos y pacientes

Sobre las reacciones de los médicos ante la búsqueda de información online por parte de sus pacientes:

- el 19,9\% de los participantes señaló que su médico reaccionó de forma indiferente;

- un 16,6\% indicó que reaccionó de forma negativa, ya sea sugiriéndole no seguir buscando, o preocupado u ofendido por la conducta del paciente;

- un 14,9\% señaló que reaccionó positivamente, ya sea porque les pareció una buena idea, o porque los motivó a seguir buscando este tipo de información.

\section{Análisis multivariable}

Para comprobar la relación de las distintas variables con la búsqueda de información online, se utilizaron regresiones lineales múltiples, y para analizar los factores que inciden en el autodiagnóstico, se emplearon regresiones binarias. La H1 plantea que los usuarios que posean un mayor grado de eficacia en el uso de la Web presentarán una mayor tendencia a buscar información en internet y a autodiagnosticarse por esta vía. La tabla 1 demuestra esta relación positiva, inclusive cuando se controla por el resto de las variables $(\beta=$ $0,114, p<0,01$ ).

Un efecto similar puede observarse en el autodiagnóstico, revelando que un punto más en la escala de eficacia está asociado a una probabilidad u odds (razón de probabilidades) de 1,65 de autodiagnosticarse, como puede observarse en la tabla 2. Con respecto a si los usuarios con mayor confianza en la información médica que encuentren online buscarán más información online sobre temas de salud, como plantea $\mathrm{H} 2$, también se corroboró que había una relación positiva entre las variables $(\beta=0,359, p<0,001)$, y ésta también se encontró en el autodiagnóstico online, ya que un punto más en la escala de confianza está asociado a una probabilidad u odds mayor del mismo de 1,76.

Es interesante destacar que la actitud de los profesionales médicos incide en las conductas de los pacientes: cuanto más positiva ha sido la experiencia con los médicos al co- 
mentarles que buscaron información online, mayor es la tendencia a seguir buscando $(\beta=0,101, p<0,05)$, pero no así con el autodiagnóstico online, lo cual corrobora parcialmente H3. Los antecedentes y preexistencias médicas también inciden en la búsqueda de información sobre salud $(\beta=0,11, p<$ 0,001 ) como pronostica $\mathrm{H} 4$, pero aquellos con condiciones de salud previas no tienden a autodiagnosticarse en mayor medida que quienes no tienen preexistencias médicas. Un efecto similar se observa en los usuarios que muestran una preocupación mayor sobre su salud $(\beta=$ $0,158, p<0,001)$, quienes a su vez también reflejan una probabilidad $u$ odds de 1,29 mayor para autodiagnóstico online, como se planteaba en $\mathrm{H} 5$.

\section{Discusión}

Este estudio exploró los factores que inciden en la búsqueda de información médica online y posterior autodiagnóstico a través de una muestra representativa de la población con acceso a internet en Chile. La investigación arrojó cuatro grandes conclusiones:

1) En la actualidad una gran parte de la población con acceso a internet busca información online sobre temas médicos y un gran porcentaje se ha autodiagnosticado por esta misma vía;

2) Las personas con mayor eficacia en el uso de la Web para temas de salud y los que tienen más confianza en estos canales tienden a buscar más información médica y también muestran una tasa mayor de autodiagnóstico;

3) La investigación determinó que:

- más de la mitad de los usuarios con acceso a internet ha conversado con sus médicos sobre lo que miran en la Red;

- el porcentaje que ha reaccionado negativamente no es significativamente mayor al que lo ha hecho de forma positiva;

- la respuesta que reciben de los médicos incide en las acciones que realizan, ya que a mayor apoyo, más alta es la frecuencia con que buscan información y se autodiagnostican.

4) Tanto los antecedentes y preexistencias médicas, como la preocupación que muestran los pacientes hacia su salud, reflejan el uso que dan a internet para temas médicos, ya que ambos tipos de usuarios son también los que más buscan información y se autodiagnostican online.

A partir de las conclusiones anteriores, habría que ahondar en dos aspectos. En primer lugar es importante entender los factores que influyen en estas conductas, como la eficacia y confianza en internet, que se relacionan de forma positiva con la tendencia a la búsqueda de salud online. El estudio comprobó que a un mayor nivel de experiencia y eficacia en la Web, es mayor la posibilidad de que el usuario reali- ce un seguimiento a temas de salud. Al parecer, la eficacia induce al usuario a otorgar un mayor grado de confianza a las informaciones que encuentra online, aunque ello no necesariamente implica que las personas con estas características dimensionen realmente los trasfondos de credibilidad y las reglas de juego de los sitios a los que acceden, ya que hay otras variables que inciden en estas conductas. Es distinto por ejemplo confiar en la información que aparece en proyectos enciclopédicos de aportación libre o foros de

Tabla 2. Regresiones logísticas para autodiagnóstico online $(N=898)$

\begin{tabular}{|c|c|c|}
\hline & \multicolumn{2}{|c|}{ Autodiagnóstico online } \\
\hline & Beta & Odds ratio \\
\hline Edad & $-0,010$ & 1,01 \\
\hline Género (1 = Mujer) & 0,338 & 0,713 \\
\hline Nivel socioeconómico & $-0,007$ & 1,02 \\
\hline$\Delta \mathrm{R}^{2}$ de Cox y Snellse & \multicolumn{2}{|c|}{1,1} \\
\hline Eficacia internet & $0,500 * * *$ & 0,606 \\
\hline Confianza internet & $0,571^{* * *}$ & 0,565 \\
\hline$\Delta R^{2}$ de Cox y Snellse & 0,059 & 16,5 \\
\hline Tiempo espera asistencia consulta & $-0,268^{* *}$ & 1,307 \\
\hline$\Delta \mathrm{R}^{2}(\%)$ & \multicolumn{2}{|c|}{0,3} \\
\hline \multicolumn{3}{|l|}{ Antecedentes médicos } \\
\hline Preocupación estado salud & $0,21^{*}$ & 0,81 \\
\hline \multicolumn{3}{|l|}{$\Delta \mathrm{R}^{2}(\%)$} \\
\hline Asertividad doctor & 0,39 & 1,039 \\
\hline \multicolumn{3}{|l|}{$\Delta \mathrm{R}^{2}(\%)$} \\
\hline Constante & \multirow{2}{*}{\multicolumn{2}{|c|}{$\begin{array}{l}2,707 \\
0,084\end{array}$}} \\
\hline $\mathrm{R}^{2}$ de Cox y Snellse & & \\
\hline
\end{tabular}

El cambio en $R^{2}$ se calcula con el $R$ de Cox y Snellse refiere a la contribución única de cada grupo de variables controlando por las variables previas ingresadas en el modelo de regresión $* \leq \leq 0,05, * * p \leq 0,01, * * * p \leq 0,001$. 
respuesta de manera confiada, que en sitios web de salud especializados.

Este trabajo permite aventurar que el usuario con mayor experiencia web comprende que la información online puede estar errada y consecuentemente filtra de mejor manera la seriedad de los sitios. Futuros estudios debieran explorar con mayor profundidad no sólo las implicaciones y efectos de esta confianza, sino también ahondar en las propiedades que afectan al grado de confianza que una persona le entrega a una plataforma web para temas de salud.

Por otra parte, es también relevante ahondar sobre la perspectiva que están tomando los médicos para responder a esta nueva realidad y cómo debiera educarse al usuario.

\section{Cuanto más positiva ha sido la experien- cia con los médicos al comentarles que buscaron información online, mayor es la tendencia a seguir buscando}

\section{Conclusión}

La búsqueda de información de salud y el autodiagnóstico online ya son una realidad en las personas con acceso a internet, y el caso chileno refleja consistentemente esta nueva realidad. Al igual que en las sociedades anglosajonas, puede ya verse una tendencia, que se observa en la cantidad de personas que obtienen en internet un mayor grado de independencia para tener información sobre su estado de salud. El actual grado de desarrollo tecnológico y su vertiginoso avance en los próximos años, no sólo modificará los alcances relativos a los tratamientos médicos sino también la manera en la que éstos llegan a la población.

Es importante entender qué perspectiva están tomando los médicos para responder a esta realidad. En el plano del personal médico, es de vital importancia introducir estos conceptos en su formación para homogeneizar las reacciones que los expertos asumen frente a esta nueva realidad. Deben ser instruidos y preparados para enfrentar al paciente 2.0: no sólo para tratarlo y sacar el mayor grado de provecho de su nueva autonomía, sino también para instruir al propio profesional de la salud sobre los alcances y las mejoras que la salud online puede sumar a la sinergia médico-paciente. En un sistema donde los tiempos de espera representan casi la totalidad de la experiencia de visita al médico, las herramientas de salud online pueden prestar servicios que alivien la presión de la limitada cantidad de consultorios médicos existentes.

Mediante el autodiagnóstico online se pueden tratar enfermedades que poseen un menor grado de urgencia, aumentando considerablemente el tiempo y la dedicación que los médicos pueden otorgar a consultas de mayor gravedad. En este artículo no se propone la ausencia del personal médico en el proceso de diagnóstico; sin embargo, se plantea que los proveedores de salud debieran considerar la incorporación de nuevas tecnologías de la información en sus prácticas, considerando que sus pacientes ya las utilizan extensivamente. La incorporación de la tecnología en el ejercicio médico podría no sólo ayudar a asegurar que la información a la que acceden los pacientes sea de calidad, sino también a descongestionar la consulta física y el sistema público de salud.

Es de suma importancia educar a la población sobre el manejo de la información que puede ser encontrada en la Web. Enseñar a distinguir los sitios que ofrecen consejos a partir de un conocimiento médico y entendido en la materia de otros que pueden terminar perjudicando la salud del paciente y generándole ansiedad. Los niveles de confianza que demuestra la población chilena en los contenidos web son altos, por lo que es necesario que los centros de salud y el sector público se encarguen de ofrecer atributos de confianza a webs que certifiquen su experiencia en temas de salud y asesoría médica. En este sentido es importante también capacitar a los profesionales del área de la salud para que sean capaces de preguntar, de ser necesario, a través de un cuestionario estandarizado en todos los centros médicos, si el paciente ha realizado alguna búsqueda de síntomas, tratamientos o enfermedades en internet. Esto con el fin de orientar y guiar la búsqueda de información de salud online hacia las webs especializadas que se proponen.

\section{Nota}

1. La Encuesta de caracterización socioeconómica nacional (Casen) es la principal encuesta que se realiza en Chile después del Censo, y se hace cada 3 años con el objetivo de obtener información para evaluar el impacto de las distintas políticas sociales.

\section{Bibliografía}

Ahmad, Farah; Hudak, Pamela L.; Bercovitz, Kim; Hollenberg, Elisa; Levinson, Wendy (2006). "Are physicians ready for patients with internet-based health information?". Journal of medical internet research, v. 8, n. 3, e22. http://dx.doi.org/10.2196/jmir.8.3.e22

Dillard, James P.; Shen, Lijiang (2005). "On the nature of reactance and its role in persuasive health communication". Communication monographs, v. 72, n. 2, pp. 144-168. http://dx.doi.org/10.1080/03637750500111815

Donnelly, Louise S.; Shaw, Rachel L.; Van-den-Akker, Olga B. (2008). "eHealth as a challenge to 'expert' power: a focus group study of internet use for health information and management". Journal of the Royal Society of Medicine, v. 101, n. 10 , pp. 501-506.

http://www.ncbi.nlm.nih.gov/pmc/articles/PMC2587202/ http://dx.doi.org/10.1258/jrsm.2008.080156

Elwyn, Glyn; Stiel, Mareike; Durand, Marie-Anne; Boivin, Jacky (2010). "The design of patient decision support interventions: addressing the theory-practice gap". Journal of evaluation in clinical practice, v. 17, n. 4, pp. 565-574. http://dx.doi.org/10.1111/j.1365-2753.2010.01517.x

Fernández-De-Mosteyrín, Sol; Del-Val-Acebrón, María; Fernández-De-Mosteyrín, Teresa; Fernández-Guerrero, Manuel (2014). "Prácticas y percepción del riesgo en hombres con infección por el virus de la inmunodeficiencia humana que tienen sexo con otros hombres". Enfermedades infecciosas y microbiología clínica, v. 32, n. 4, pp. 219-224. 
http://dx.doi.org/10.1016/j.eimc.2013.04.017

Fernández-Martínez, Rafael; Fernández-Rodríguez, Concepción (2001). "Factores asociados a preocupación hipocondríaca en pacientes de un centro de salud de atención primaria". Psicothema, v. 13, n. 4, pp. 659-670.

http://www.psicothema.com/psicothema. asp? $\mathrm{id}=494$

Finch, Tracy L.; Mort, Maggie; Mair, Frances S.; May, Carl R. (2008). "Future patients? Telehealthcare, roles and responsibilities". Health \& social care in the community, v. 16, n. 1, pp. 86-95.

http://dx.doi.org/10.1111/j.1365-2524.2007.00726.x

Fox, Susannah; Duggan, Maeve (2013). "Health online 2013". Pew internet and American life project. Washington, DC. http://www.pewinternet.org/2013/01/15/health-online-2013

Hornik, Robert; Parvanta, Sarah; Mello, Susan; Freres, Derek; Kelly, Bridget; Schwartz, J. Sanford (2013). "Effects of scanning (routine health information exposure) on cancer screening and prevention behaviors in the general population". Journal of health communication, v. 18, n. 12, pp. 1422-1435.

http://www.ncbi.nlm.nih.gov/pmc/articles/PMC4235954/ http://dx.doi.org/10.1080/10810730.2013.798381

Lowrey, Wilson; Anderson, William B. (2006). "The impact of internet use on the public perception of physicians: a perspective from the sociology of professions literature". Health communication, v. 19, n. 2, pp. 125-131.

http://dx.doi.org/10.1207/s15327027hc1902_4

Mair, Frances S.; May, Carl; O’Donnell, Catherine; Finch, Tracy; Sullivan, Frank; Murray, Elisabeth (2012). "Factors that promote or inhibit the implementation of e-health systems: an explanatory systematic review". Bulletin of the World Health Organization, v. 90, n. 5, pp. 357-364.

http://dx.doi.org/10.2471/BLT.11.099424

Mira-Solves, José-Joaquín; Llinás-Santacreu, Gilberto; Lorenzo-Martínez, Susana; Pérez-Jover, Virtudes (2010). "Preguntas más frecuentes sobre repercusión de la e-salud en la relación entre médico y paciente". Atención primaria, v. 42, n. 2, pp. 112-114.

http://www.elsevier.es/es-revista-atencion-primaria-27articulo-preguntas-mas-frecuentes-repercusion-e-saludrelacion-medico-13146919

Niederdeppe, Jeff; Hornik, Robert C.; Kelly, Bridget J.; Frosch, Dominick L.; Romantan, Anca; Stevens, Robin S.; Barg, Frances K.; Weiner, Judith L.; Schwartz, Judith S.
(2007). "Examining the dimensions of cancer-related information seeking and scanning behavior". Health communication, v. 22, n. 2, pp. 153-167.

http://dx.doi.org/10.1080/10410230701454189

Nijland, Nicol; Van-Gemert-Pijnen, Julia; Boer, Henk; Steehouder, Michaël F.; Seydel, Erwin R. (2008). "Evaluation of internet-based technology for supporting self-care: Problems encountered by patients and caregivers when using self-care applications". Journal of medical internet research, v. 10, n. 2.

http://dx.doi.org/10.2196/jmir.957

Norman, Cameron D.; Skinner, Harvey A. (2006). "eHeals: The eHealth literacy scale". Journal of medical internet research, v. 8, n. 4.

http://dx.doi.org/10.2196/jmir.8.4.e27

Pastorelli, Romina-Vanesa; Bañón-González, Sara-María; Trigo-Campoy, Amanda; Martín-Carballeda, Julia; González-Vallejo, José-Víctor (2011). "Trastorno hipocondríaco". Revista psiquiatría.com, v. 15, pp. 1-26.

Rice, Ronald E. (2006). "Influences, usage, and outcomes of internet health information searching: multivariate results from the Pew surveys". International journal of medical informatics, v. 75, n. 1, pp. 8-28.

http://dx.doi.org/10.1016/j.ijmedinf.2005.07.032

Smith, Peter; Fox, Adam T.; Davies, Patrick; Hamidi-Manesh, Laila (2006). "Cyberchondriacs". International journal of adolescent medicine and health, v. 18, n. 2, pp. 209-214. http://dx.doi.org/10.1515/IJAMH.2006.18.2.209

Subtel (2013). Informe series estadísticas: primer semestre 2013. Subsecretaría de Telecomunicaciones. Unidad de Estudios y Planificación Estratégica. Santiago de Chile.

http://www.subtel.gob.cl/images/stories/apoyo_articulos/ notas_prensa/informe_seriesq2_2013_vfinal.pdf

Sundar, S. Shyam; Knobloch-Westerwick, Silvia; Hastall, Matthias R. (2007). "News cues: Information scent and cognitive heuristics". Journal of the American Society for Information Science and Technology, v. 58, n. 3, pp. 366-378. http://dx.doi.org/10.1002/asi.20511

Weinhold, Ines; Gastaldi, Luca (2015). "From shared decision making to patient engagement in health care processes: The role of digital technologies". En: Gurtner, Sebastian; Soyez, Katja (eds.). Challenges and opportunities in health care management. Springer, pp. 185-196. ISBN: 9783319 121789 


\section{Colección de libros de bolsillo}

\section{El profesional de la información (Editorial UOC)}
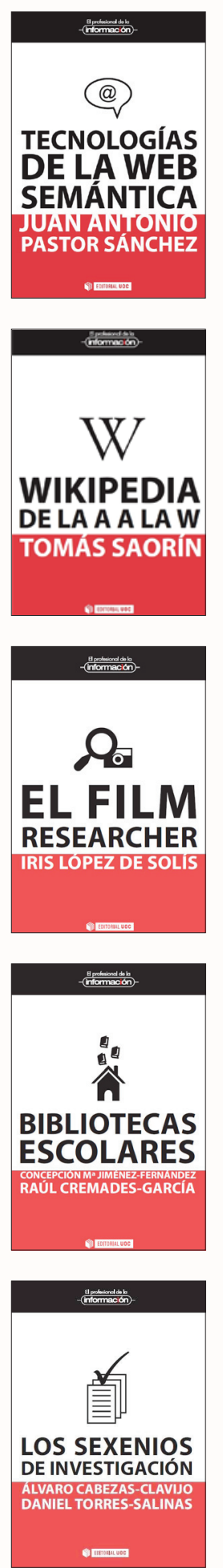
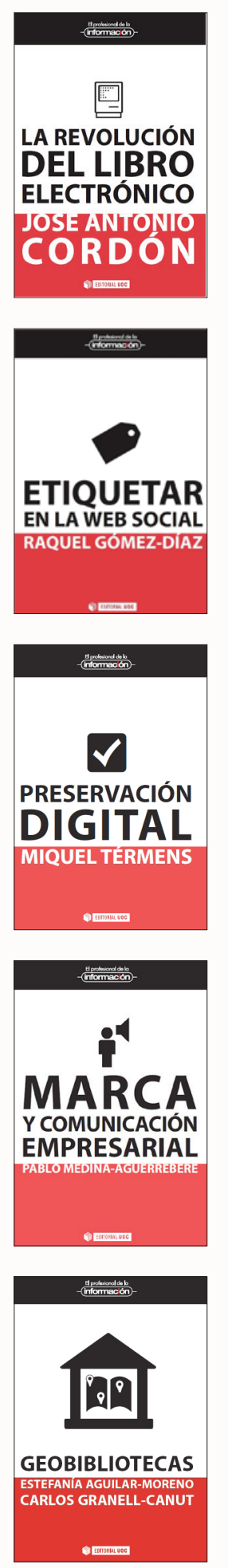
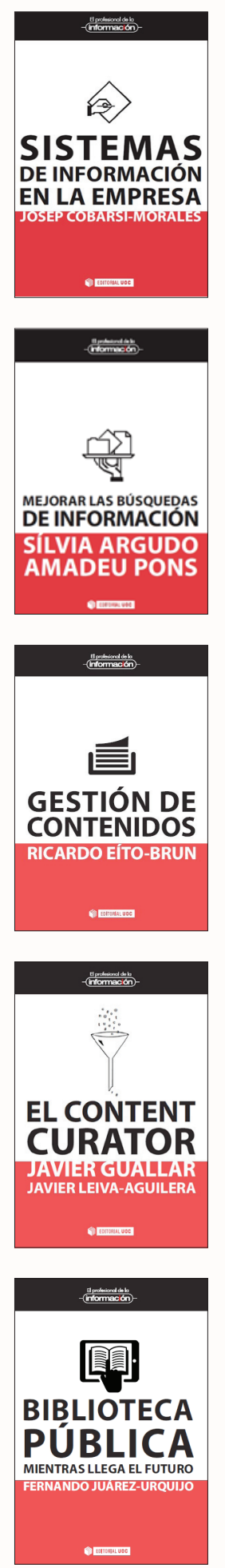
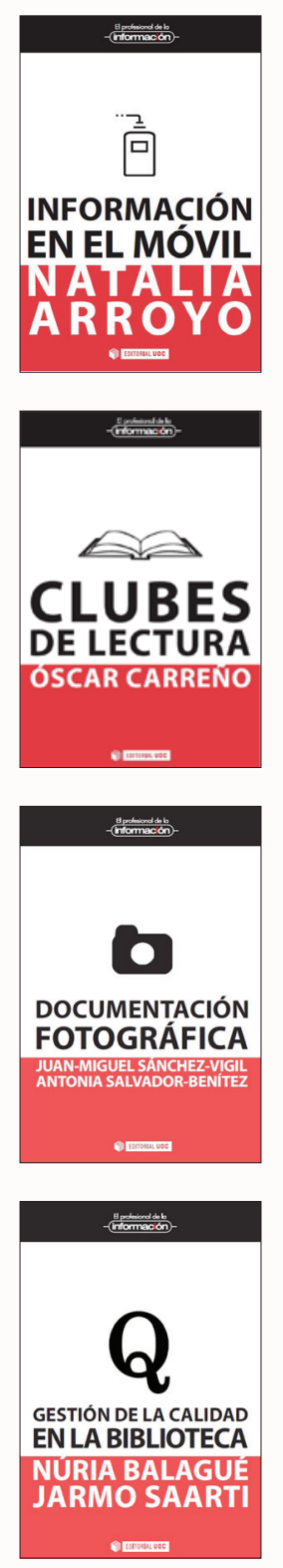
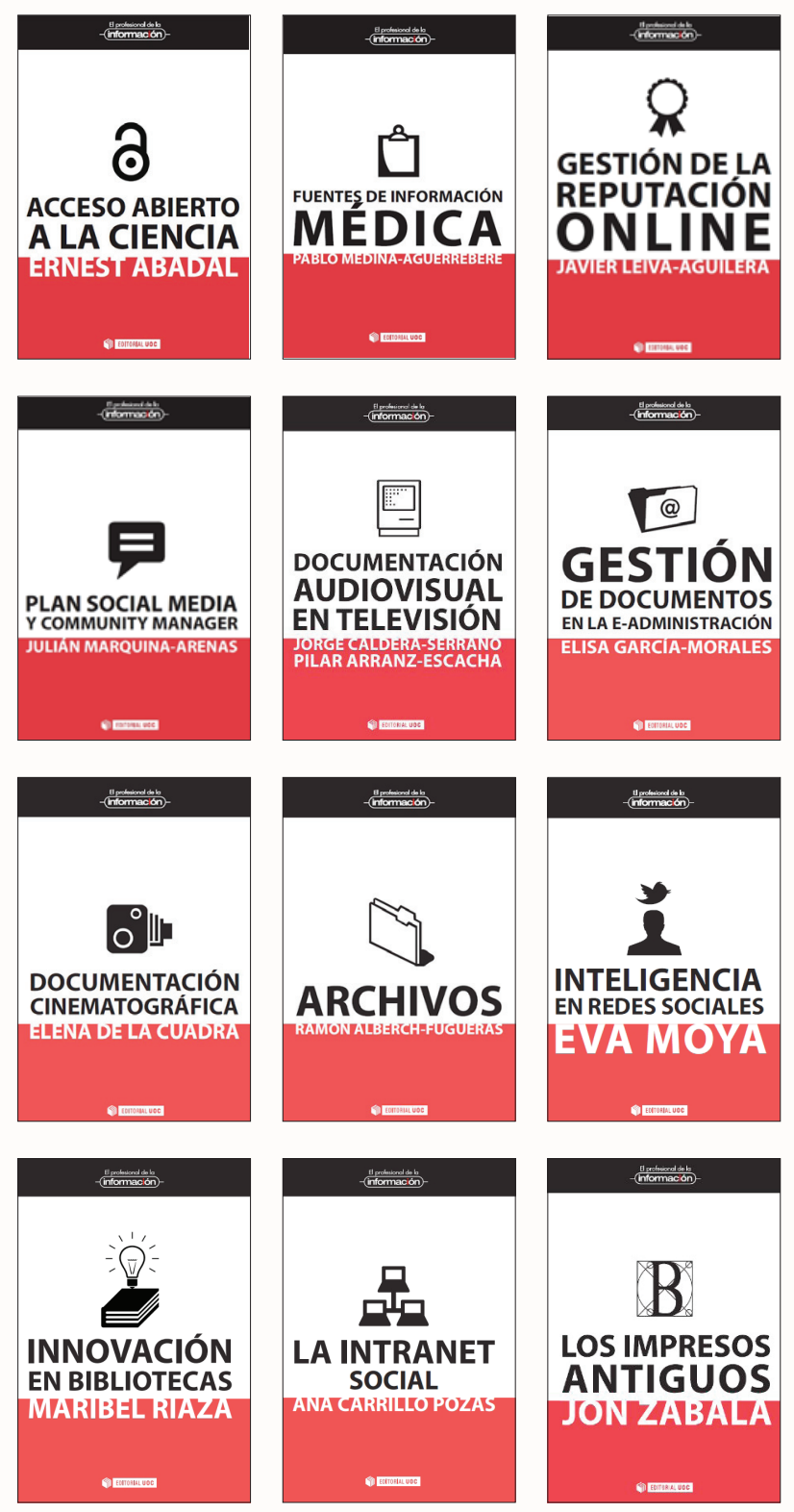

El profesional de la -información

STI EDITORIAL UOC

Más información: 